\title{
Curvas de Peso al Nacer
}

\section{Birth-weight curves}

\author{
Nora E. Montoya-Restrepo ${ }^{1}$ y Juan C. Correa-Morales²
}

1. Susalud. Medellín, Colombia.noramore@susalud.com.co

2. Escuela de Estadística, Universidad Nacional de Colombia, Sede Medellín. jccorrea@unalmed.edu.co

Recibido 8 Junio 2006/Enviado para Modificación 12 Noviembre 2006/Aceptado 12 Enero 2007

\section{RESUMEN}

Objetivo Elaborar curvas que relacionen el peso al nacer y edad gestacional, y sirvan de estándar para la población de recién nacidos en Colombia.

Métodos Las curvas fueron elaboradas utilizando los pesos de los recién nacidos producto de embarazos que finalizaron entre las semanas 22 y 44 . Se elaboraron tres tablas con sus respectivas figuras, utilizando regresiones monótonas ajustadas sobre los percentiles muestrales calculados para los diferentes estratos definidos por las covariables sexo y número de semanas.

Resultados Se construyeron las curvas porcentuales para el peso de los neonatos a los niveles $5,10,25,50,75,90$ y $95 \%$, además de las tablas con sus valores. Conclusiones Disponer de curvas de peso al nacer elaboradas con los datos de los recién nacidos pertenecientes a las poblaciones de las cuales provienen, permite una mejor clasificación de los recién nacidos.

Palabras Clave: Curvas de peso, peso al nacer, edad gestacional (fuente: DeCS, BIREME).

\section{ABSTRACT}

Objective This work was aimed at constructing curves relating birth-weight to gestational age; these can be used as a standard for the newborn in Colombia.

Method The curves were drawn up using birth-weights from pregnancies lasting 2244 weeks. Three tables were drawn up; these are presented, along with their respective graphs. They were constructed using monotonic regression from the sample percentiles computed in strata defined by gender and the number of weeks.

Results Percentile curves were built for 5, 10, 25, 50, 75, 90 and 95\% percentiles for the newborn in the form of tables and graphs.

Conclusions Constructing birth-weight curves from data pertaining to the target population led to better classification of the newborn.

Key Words: Birth-weight, birth-weight curve, gestational age (source: MeSH, NLM) 
$\mathrm{E}$ l peso del recién nacido se ha constituido en una de las variables predictoras de la morbilidad y la mortalidad infantil. Cuanto menor es el peso, mayor es la probabilidad de morir durante el primer año de vida, siendo el bajo peso alnacer el mayor determinante de la mortalidad en este grupo poblacional y el responsable del $66 \%$ de todas las muertes neonatales (1). Una de las variables antropométricas más utilizadas para evaluar el crecimiento fetal es el peso al nacer y, una tabla que relacione éste con la edad gestacional en ese momento, permite la clasificación de los recién nacidos según los percentiles, por ejemplo: recién nacidos grandes, los que se encuentran por encima del percentil 90 o recién nacidos pequeños, los que se hallan por debajo del percentil 10 (2).

Tanto los recién nacidos grandes para la edad gestacional como los pequeños, son considerados poblaciones de alto riesgo para presentar problemas de morbilidad y mortalidad. Entre los recién nacidos grandes los problemas más comunes son los traumatismos fetales y los trastornos del metabolismo (2); en los recién nacidos pequeños, lo son las afecciones perinatales, las anomalías congénitas y las enfermedades infecciosas (3). Las tasas de mortalidad entre los recién nacidos pequeños son de cinco a seis veces mayores que las que presentan los neonatos con crecimiento normal para la misma edad gestacional (2).

En la década de los sesenta se publicaron los trabajos de Lubchenco y colaboradores sobre el crecimiento intrauterino y, en los setenta los de Babson y colaboradores, y sus resultados se convirtieron en estándares utilizados a nivel mundial. De igual manera, Lejarraga en Buenos Aires, Cascante en Costa Rica y González en Chile (4), entre otros, aportaron resultados similares a los anteriores. Sin embargo, se ha visto que el empleo de patrones extranjeros subestima las condiciones de las poblaciones en las que se está evaluando el neonato problema, por lo cual se hace necesario disponer de curvas propias evitando, de esta manera, extrapolar datos de unas regiones a otras.

En Colombia, no conocemos que se hayan construido curvas que relacionen peso y edad gestacional al momento de nacer, por esta razón, el objetivo del presente trabajo es elaborar curvas que sirvan de estándar para la población de neonatos. 


\section{MÉTODOS}

Para elaborar las curvas de peso al nacer según la edad gestacional, se utilizaron los datos sobre el peso de los recién nacidos afiliados al régimen contributivo de la empresa promotora de salud Susalud, correspondientes al período Enero de 1999 a Diciembre de 2005. En la base de datos se incluyeron los pesos de los recién nacidos producto de embarazos que finalizaron entre las semanas 22 y 44 . Se disponía de los datos de 58406 nacimientos ocurridos en la red de instituciones prestadoras de servicios de salud de segundo y tercer nivel de atención, encargadas de atender los partos de las afiliadas en las ciudades de Medellín, Cali, Bogotá y Barranquilla pero, al depurar la base de datos se excluyeron los registros en los cuales el peso era menor de 500 gramos, aquellos en los que no se conocía la edad gestacional, los fallecidos, registros de gestantes con hipertensión o diabetes, niños con malformaciones o productos de partos múltiples, lo cual dio como resultado final un total de 54044 registros.

La edad gestacional fue estimada de acuerdo a lo estipulado en el Protocolo del Control del Proceso Reproductivo (5) de la empresa promotora de salud Susalud, esto es, por el examen físico del recién nacido utilizando el método de Capurro, por la fórmula Naegele o por ecografía y, el peso al nacer fue tomado como el peso en gramos del niño desnudo tomado inmediatamente después del expulsivo, el cual fue determinado por personal médico o paramédico adecuadamente entrenado y utilizando para ello balanzas mecánicas o digitales calibradas.

La base de datos inicial fue elaborada en Excel 97 y la tabulación y el procesamiento de la información se realizó utilizando el paquete estadístico $\mathrm{R}$ (6).

Con estos datos se elaboraron las tablas con sus respectivos gráficos, utilizando regresiones monótonas ajustadas sobre los percentiles muestrales calculados para los diferentes estratos definidos por las covariables sexo y número de semanas (7).

\section{RESULTADOS}

Para elaborar las curvas se contó con un total de 54044 datos sobre los pesos de recién nacidos; de éstos, el 47,5 \% pertenecían al género femenino y 52,5 $\%$ al género masculino. 
La edad promedio de las gestantes fue de 26,8 años, el 50 \% de ellas tenían una edad menor o igual a 26 años, el 75 \% edad igual o inferior a 31 años, la edad mínima fue 10 años y la máxima 51 años.

La mediana del peso de todos los recién nacidos fue de 3120 gramos, siendo igual a 3200 gramos para los niños y a 3100 gramos para las niñas (peso que fue 3,1\% menor que el de los niños). En cuanto a la edad gestacional, el $25 \%$ de las gestantes presentaban en el momento del nacimiento una edad gestacional menor o igual a 38 semanas, el 50\% menor o igual a 39 y el $75 \%$ menor o igual a 40 semanas.

Con los valores de los pesos para cada una de las semanas de gestación, se calcularon los percentiles 5, 10, 25, 50, 75, 90 y $95 \%$, tanto a nivel general para ambos géneros (Tabla 1), como para cada uno de ellos por separado (Tablas 2 y 3) y, posteriormente, con estos datos fueron elaboradas las gráficas de las curvas que relacionan el peso al nacer y la semana de gestación (Figuras 1,2,3).

Tabla 1. Peso en gramos al nacer para ambos géneros Enero 1999 a Diciembre de 2005

\begin{tabular}{|c|c|c|c|c|c|c|c|}
\hline Semana & p5 & p10 & p25 & p50 & p75 & P90 & p95 \\
\hline 22 & 543 & 575 & 601 & 601 & 692 & 768 & 775 \\
\hline 23 & 602 & 602 & 642 & 686 & 764 & 862 & 874 \\
\hline 24 & 624 & 630 & 668 & 735 & 894 & 1012 & 1027 \\
\hline 25 & 644 & 676 & 722 & 742 & 1048 & 1301 & 1360 \\
\hline 26 & 653 & 703 & 796 & 901 & 1067 & 1670 & 2311 \\
\hline 27 & 723 & 775 & 914 & 1041 & 1175 & 1855 & 2529 \\
\hline 28 & 750 & 853 & 1003 & 1164 & 1501 & 2296 & 2557 \\
\hline 29 & 1000 & 1050 & 1180 & 1400 & 1601 & 2462 & 2704 \\
\hline 30 & 1002 & 1051 & 1240 & 1446 & 1721 & 2503 & 2896 \\
\hline 31 & 1182 & 1262 & 1515 & 1750 & 1950 & 2600 & 2925 \\
\hline 32 & 1239 & 1419 & 1600 & 1850 & 2050 & 2735 & 2977 \\
\hline 33 & 1381 & 1612 & 1807 & 2034 & 2398 & 2839 & 3053 \\
\hline 34 & 1641 & 1796 & 2047 & 2319 & 2637 & 3033 & 3291 \\
\hline 35 & 1851 & 1985 & 2259 & 2529 & 2837 & 3096 & 3323 \\
\hline 36 & 2049 & 2229 & 2458 & 2699 & 2997 & 3236 & 3486 \\
\hline 37 & 2285 & 2441 & 2648 & 2898 & 3176 & 3416 & 3591 \\
\hline 38 & 2485 & 2593 & 2838 & 3098 & 3317 & 3567 & 3719 \\
\hline 39 & 2592 & 2694 & 2948 & 3199 & 3427 & 3676 & 3835 \\
\hline 40 & 2690 & 2794 & 3013 & 3289 & 3527 & 3773 & 3936 \\
\hline 41 & 2728 & 2800 & 3000 & 3253 & 3437 & 3674 & 3791 \\
\hline 42 & 2738 & 2883 & 3097 & 3328 & 3504 & 3742 & 3887 \\
\hline 43 & 3111 & 3152 & 3168 & 3348 & 3564 & 3800 & 3967 \\
\hline 44 & 3168 & 3170 & 3238 & 3358 & 3591 & 3828 & 3991 \\
\hline
\end{tabular}


Tabla 2. Peso en gramos al nacer para el género femenino Enero 1999 a Diciembre de 2005

\begin{tabular}{|c|c|c|c|c|c|c|c|}
\hline \multirow[b]{2}{*}{ Semana } & \multicolumn{7}{|c|}{ Percentiles } \\
\hline & p5 & p10 & p25 & p50 & p75 & p90 & p95 \\
\hline 22 & 525 & 540 & 550 & 569 & 603 & 647 & 707 \\
\hline 23 & 553 & 557 & 627 & 723 & 757 & 792 & 834 \\
\hline 24 & 649 & 675 & 740 & 747 & 806 & 858 & 878 \\
\hline 25 & 696 & 740 & 743 & 793 & 833 & 875 & 889 \\
\hline 26 & 740 & 745 & 788 & 840 & 910 & 1120 & 1359 \\
\hline 27 & 749 & 759 & 931 & 1052 & 1150 & 1709 & 2118 \\
\hline 28 & 856 & 905 & 955 & 1122 & 1360 & 2050 & 2565 \\
\hline 29 & 926 & 987 & 1181 & 1402 & 1614 & 2160 & 2634 \\
\hline 30 & 965 & 1051 & 1198 & 1437 & 1740 & 2241 & 2738 \\
\hline 31 & 1190 & 1257 & 1409 & 1594 & 1960 & 2373 & 2988 \\
\hline 32 & 1212 & 1404 & 1582 & 1901 & 2124 & 2471 & 3031 \\
\hline 33 & 1352 & 1430 & 1765 & 2000 & 2273 & 2685 & 3109 \\
\hline 34 & 1597 & 1733 & 1997 & 2276 & 2688 & 3038 & 3165 \\
\hline 35 & 1892 & 2001 & 2244 & 2561 & 2889 & 3150 & 3351 \\
\hline 36 & 1996 & 2136 & 2394 & 2656 & 2991 & 3169 & 3393 \\
\hline 37 & 2266 & 2408 & 2609 & 2886 & 3106 & 3226 & 3468 \\
\hline 38 & 2430 & 2590 & 2788 & 2993 & 3163 & 3361 & 3562 \\
\hline 39 & 2551 & 2693 & 2883 & 3091 & 3278 & 3471 & 3643 \\
\hline 40 & 2624 & 2755 & 2899 & 3163 & 3364 & 3573 & 3736 \\
\hline 41 & 2693 & 2778 & 2990 & 3196 & 3472 & 3677 & 3802 \\
\hline 42 & 2757 & 2837 & 3035 & 3200 & 3500 & 3736 & 3834 \\
\hline 43 & 3118 & 3134 & 3153 & 3285 & 3500 & 3749 & 3910 \\
\hline 44 & 3220 & 3228 & 3247 & 3300 & 3555 & 3755 & 3943 \\
\hline
\end{tabular}

Así mismo, aunque no era un objetivo del estudio, se elaboró la gráfica que muestra la probabilidad de bajo peso al nacer (recién nacido cuyo peso es menor de 2500 gramos) de acuerdo a las semanas de gestación en la población de estudio (Figura 4).

\section{DISCUSIÓN}

Ante la imposibilidad de disponer de curvas elaboradas con datos de fetos sanos que relacionen el peso con la edad gestacional en el útero a lo largo de toda la gestación, curvas que en un futuro se podrán elaborar mediante la ultrasonografía, desde la década de los sesenta se han construido curvas con 
los datos obtenidos de los recién nacidos a diferentes edades gestacionales (8).

Debido a que las características socioeconómicas, ecológicas y étnicas de una población pueden influir en los patrones de crecimiento de la misma, la Organización Mundial de la Salud (OMS) recomendó que el crecimiento intrauterino de un recién nacido perteneciente a una población específica, sea valorado por comparación con estándares obtenidos de la misma población mediante estudios prospectivos (4).

Las presentes curvas no son el producto de un estudio prospectivo, sin embargo, al depurar la base de datos inicial se eliminaron los registros en los cuales el peso del recién nacido podría estar afectado: los hijos de madres con hipertensión o diabetes, los recién nacidos muertos o con malformaciones y los productos de embarazos múltiples; por esta razón, y teniendo en cuenta que el número de registros utilizados para este estudio fue alrededor de 54 000 (las curvas de Lubchenco y colaboradores ampliamente utilizadas a nivel mundial están basadas en 5635 nacimientos ocurridos entre 1948 y 1961 en Denver, Estados Unidos (4), consideramos que ellas pueden ser apropiadas para evaluar a nuestros recién nacidos al compararlos con patrones obtenidos de las poblaciones a las cuales pertenecen y, de igual manera, creemos que éstas pueden llegar a ser un valioso instrumento tanto para el neonatólogo como para el obstetra, pues los datos provienen de una población cuyas características demográficas son similares a las de la población colombiana, esto es, una población donde alrededor del $30 \%$ son mujeres de 15 a 49 años, donde el grupo de mujeres que presenta la tasa de fecundidad más alta es el de 20 a 24 años y donde las dos terceras partes de ellas tienen ingresos económicos inferiores a dos salarios mínimos legales vigentes (11).

Estamos seguros que estas curvas pueden ser mejoradas con la inclusión de otras variables que pueden afectar el crecimiento fetal, variables a las que no fue fácil acceder pues en una gran proporción de los registros se hallaban en forma de texto o en formato impreso lo cual hizo difícil su extracción siendo esta la mayor limitante del estudio; entre estas variables se encuentran: antecedentes de ruptura prematura de membranas, tres o más embarazos anteriores, abortos previos, intervalo intergenésico menor de 2 años, consumo de alcohol o cigarrillo durante el embarazo, primer control tardío del embarazo (después de la semana 22), nivel socioeconómico y educativo de la madre, la altura sobre el nivel del mar $(8,9,10)$, factores que influyen en el desarrollo fetal y los cuales hacen que durante todo el periodo de gestación, aún después 
de la semana 37, exista la probabilidad de que el recién nacido presente bajo peso al nacer (peso menor de 2500 gramos), como se puede observar en la Figura 4.

De igual manera recomendamos la realización de estudios que permitan validar las curvas que aquí presentamos, estudios en los cuales se compare la clasificación de los recién nacidos usando las tablas que han sido utilizados como estándares a nivel mundial y las nuestras, para, de esta manera, cuantificar las diferencias en la clasificación de los recién nacidos utilizando ambos patrones

Tabla 3. Peso en gramos al nacer para el género masculino Enero 1999 a Diciembre de 2005

\begin{tabular}{|c|c|c|c|c|c|c|c|}
\hline \multirow[b]{2}{*}{ Semana } & \multicolumn{7}{|c|}{ Percentiles } \\
\hline & p5 & $\mathrm{p} 10$ & p25 & p50 & p75 & p90 & P95 \\
\hline 22 & 518 & 535 & 551 & 576 & 653 & 731 & 749 \\
\hline 23 & 532 & 545 & 572 & 602 & 685 & 829 & 891 \\
\hline 24 & 544 & 566 & 631 & 701 & 772 & 883 & 993 \\
\hline 25 & 549 & 590 & 674 & 734 & 814 & 983 & 1052 \\
\hline 26 & 605 & 641 & 702 & 945 & 1047 & 1279 & 1379 \\
\hline 27 & 666 & 702 & 794 & 953 & 1098 & 1711 & 2008 \\
\hline 28 & 756 & 800 & 991 & 1181 & 1472 & 1865 & 2319 \\
\hline 29 & 835 & 922 & 1052 & 1292 & 1508 & 2246 & 2477 \\
\hline 30 & 970 & 1037 & 1200 & 1489 & 1656 & 2323 & 2630 \\
\hline 31 & 1141 & 1201 & 1475 & 1750 & 1914 & 2512 & 2842 \\
\hline 32 & 1241 & 1370 & 1558 & 1865 & 2107 & 2689 & 2960 \\
\hline 33 & 1384 & 1508 & 1800 & 2010 & 2338 & 2895 & 2972 \\
\hline 34 & 1599 & 1799 & 1999 & 2299 & 2594 & 2993 & 3229 \\
\hline 35 & 1798 & 1978 & 2258 & 2539 & 2793 & 3133 & 3381 \\
\hline 36 & 2015 & 2197 & 2458 & 2738 & 3028 & 3276 & 3430 \\
\hline 37 & 2256 & 2397 & 2677 & 2948 & 3189 & 3405 & 3517 \\
\hline 38 & 2500 & 2675 & 2896 & 3118 & 3384 & 3483 & 3656 \\
\hline 39 & 2598 & 2797 & 2998 & 3199 & 3418 & 3613 & 3780 \\
\hline 40 & 2697 & 2849 & 3078 & 3298 & 3491 & 3726 & 3888 \\
\hline 41 & 2709 & 2899 & 3099 & 3378 & 3588 & 3822 & 3970 \\
\hline 42 & 2739 & 2900 & 3100 & 3400 & 3600 & 3871 & 4000 \\
\hline 43 & 3116 & 3151 & 3245 & 3400 & 3602 & 3888 & 4093 \\
\hline
\end{tabular}


Figura 1. Curvas de peso al nacer para ambos géneros

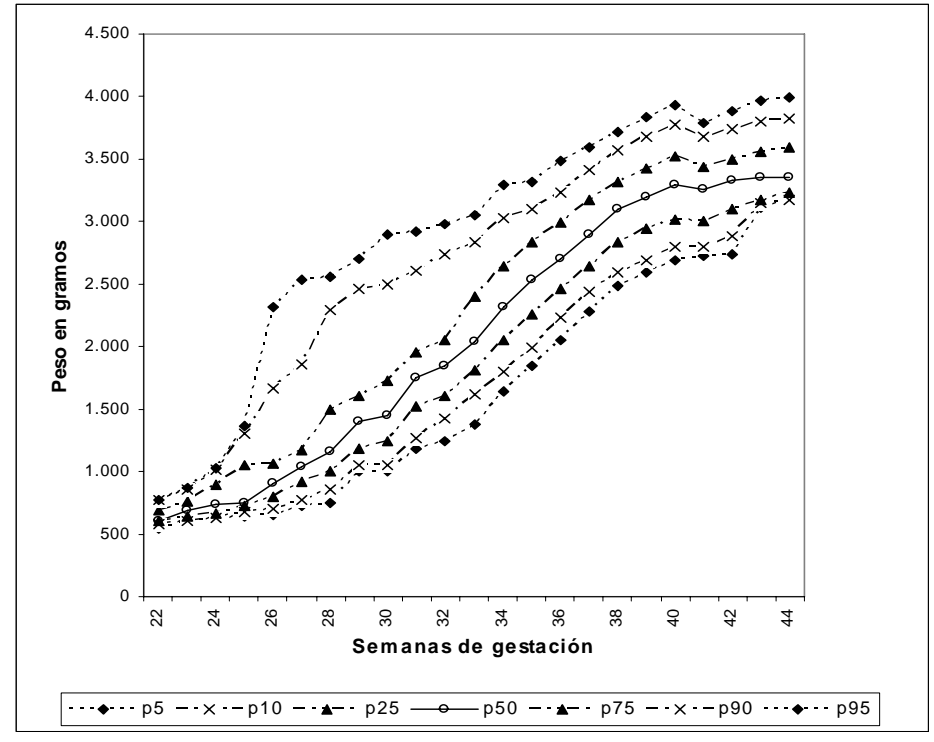

Figura 2. Curvas de peso al nacer para el género femenino

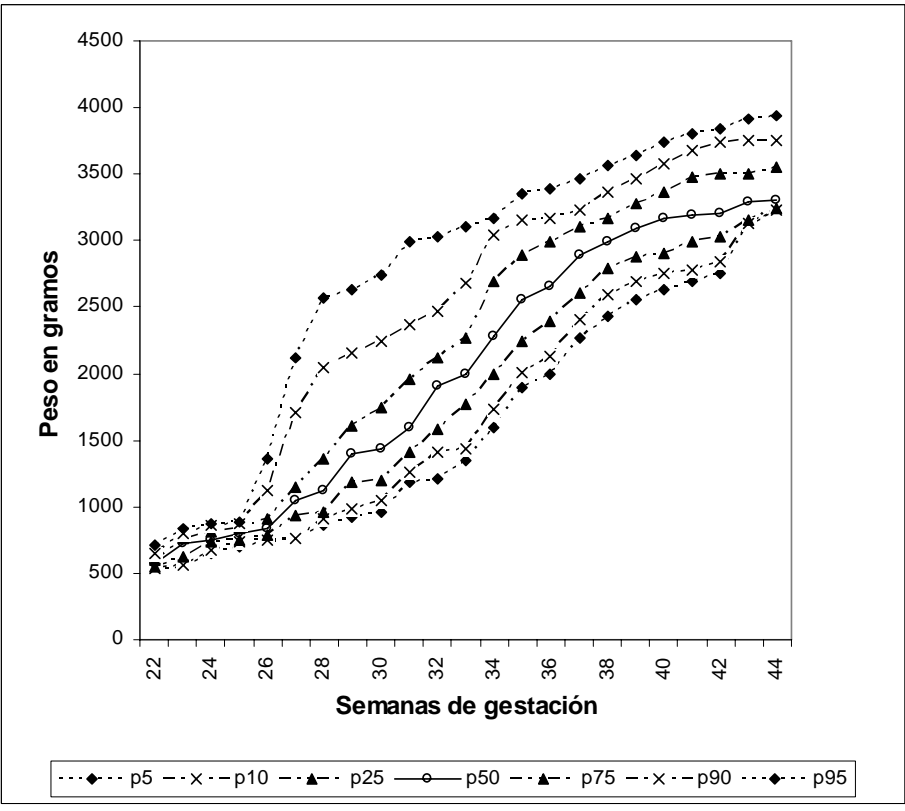


Figura 3. Curvas de peso al nacer para el género masculino

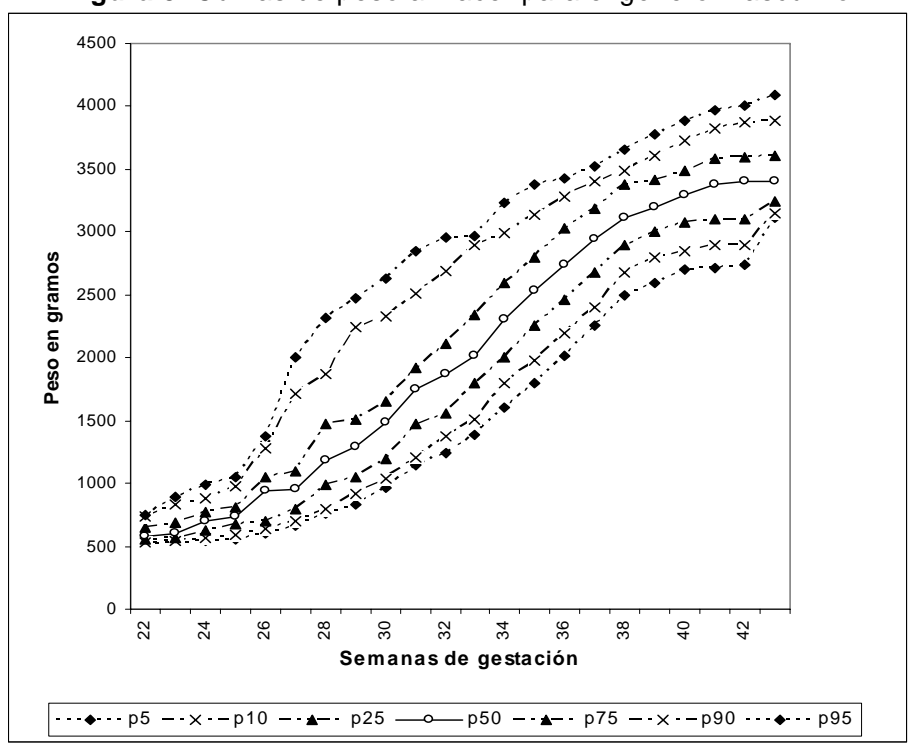

Figura 4. Probabilidad de bajo peso al nacer (BPN) por semana de gestación

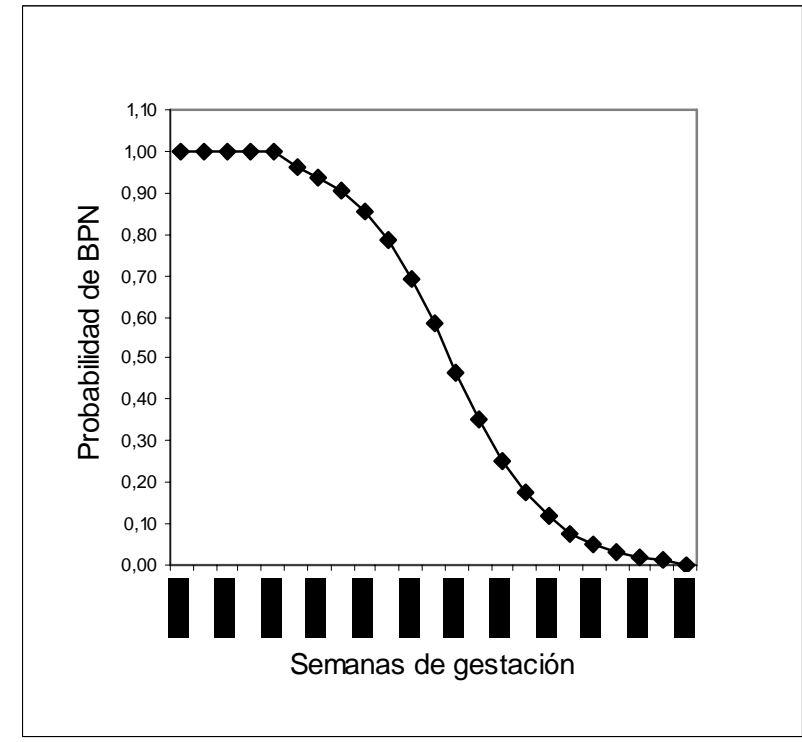


Agradecimientos. Agradecemos la colaboración de la Compañía Suramericana de Servicios de Salud S.A. Susalud, por permitir la utilización de la base de datos con los cuales fue realizado este trabajo y autorizar la publicación de los resultados.

\section{REFERENCIAS}

1. Mariotoni G, Barros A. Peso ao nacer e mortalidade hospitalar entre nacidos vivos, 1975-1996. Revista de Saúde Pública 2000; 34: 71-76.

2. Morales V, Lacarrubba J, Rotela GJ, Acosta A. Curvas estándares de peso al nacimiento para neonatos del Paraguay. Arch. argent. pediatr 2000; 98:376-381.

3. Abeya E. Mortalidad infantil de niños de bajo peso al nacer. Arch. argent. pediatr 2001; 99:7.

4. González R, Gómez R, Castro R, Nien JK, Merino P, Etchegaray A y cols. Curva nacional de distribución de peso al nacer según edad gestacional. Chile, 1993 a 2000. Rev Med Chile 2004; 132: 1155-1165. [en línea] nov. 2006, [fecha de acceso 15 Noviembre 2006].

Disponibleen: http://www.scielo.cl/scielo.php?script=sci_arttext\&pid=S003498872004001000001\&lng=es\&nrm=iso.

5. Jubiz A, Bustamente N, Gómez A. Protocolo del control del proceso reproductivo. Medellín: SUSALUD; 1999.

6. A Language and Environment for Statistical Computing, R Development Core Team. R Foundation for Statistical Computing. Vienna, Austria, 2006, [en línea] nov 2006, [fecha de acceso 16 Noviembre de 2006 ]. Disponible en: http://www.Rproject.org.-0.

7. Conover WJ. Practical Nonparametric Statistics. Third Ed. New York: John Wiley \& Sons; 1999.

8. Fescina RH, Schwarcz R. Crecimiento intrauterino. La mujer gestante. En: Cuminsky M, Moreno EM, Suárez. Crecimiento y Desarrollo(Ed.), Publicación Científica No 510. Washington, DC: OPS; 1988; p. 71-89.

9. Fescina RH, Schwarcz R, Díaz AG. Vigilancia del Crecimiento Fetal. Publicación Científica No 1261. Washington DC: Centro Latinoamericano de Perinatología y Desarrollo Humano; 1996. p. 9-10.

10.Grau M, Saenz L y Cabrales J. Factores de riesgo del bajo peso al nacer, Hospital Gineco-Obstétrico Provincial de Sancti Spíritus, Cuba. Rev Panam Salud Publica vol.6 n.2 Washington Aug. 1999. [en línea] nov 2006, [fecha de acceso 17 Noviembre de 2006]. Disponible en: http://www.scielosp.org/ scielo.php?script=sci_arttext \&pid=S1020 $-49891999000700003 \& \operatorname{lng}=$ en\&nrm=iso

11. Montoya N. La salud de nuestros afiliados al POS 2004. Situación de salud y características de la población. Susalud. Ed. Litotipo. Julio 2005. 